\title{
A Summative Comparison of Blind Channel Estimation Techniques for Orthogonal Frequency Division Multiplexing Systems
}

\author{
Vivek Kumar Gupta ${ }^{1}$, Sandip Vijay \\ ${ }^{1}$ Uttarakhand Technical University, India \\ ${ }^{2}$ ICFAI University, India
}

\begin{tabular}{l} 
Article Info \\
\hline Article history: \\
Received Jun 9, 2016 \\
Revised Nov 20, 2016 \\
Accepted Dec 11, 2016 \\
\hline
\end{tabular}

Keyword:

Blind, semi blind and trained channel estimation

LMSE-least mean square error Maximum likelihood MSE-Mean square error OFDM-Orthogonal frequency division multiplexing SDR-semi definite approach SDR-semi definite relaxation

\begin{abstract}
The OFDM technique i.e. Orthogonal frequency division multiplexing has become prominent in wireless communication since its instruction in 1950's due to its feature of combating the multipath fading and other losses. In an OFDM system, a large number of orthogonal, overlapping, narrow band subchannels or subcarriers, transmitted in parallel, divide the available transmission bandwidth. The separation of the subcarriers is theoretically optimal such that there is a very compact spectral utilization. This paper reviewed the possible approaches for blind channel estimation in the light of the improved performance in terms of speed of convergence and complexity. There were various researches which adopted the ways for channel estimation for Blind, Semi Blind and trained channel estimators and detectors. Various ways of channel estimation such as Subspace, iteration based, LMSE or MSE based (using statistical methods), SDR, Maximum likelihood approach, cyclostationarity, Redundancy and Cyclic prefix based. The paper reviewed all the above approaches in order to summarize the outcomes of approaches aimed at optimum performance for channel estimation in OFDM systems.
\end{abstract}

Copyright (c) 2018 Institute of Advanced Engineering and Science. All rights reserved.

\section{Corresponding Author:}

Vivek Kumar Gupta,

Uttarakhand Technical University, Dehradun, Uttarakhand, India.

Email:vivekgupta79ster@gmail.com

\section{INTRODUCTION}

In an OFDM system, a large number of orthogonal, overlapping, narrow band sub-channels or subcarriers, transmitted in parallel, divide the available transmission bandwidth. The separation of the subcarriers is theoretically optimal such that there is a very compact spectral utilization. In this paper first the Introduction to an OFDM system is taken in brief and there after different method used to obtain the best performance for Blind channel estimation are analyzed with a view to present a summarized form of outcomes of the various channel estimation techniques with reference to Blind ,Semi Blind and Trained channel estimators and detectors.

\section{RESEARCH METHOD}

With the rapid growth of digital communication in recent years, the need for high speed data transmission is increased. Moreover, future wireless systems are expected to support a wide range of services which includes video, data and voice. Orthogonal Frequency Division Multiplexing (OFDM) is a promising candidate for achieving high data rates in mobile environment because of its multicarrier modulation technique.In an OFDM system, a large number of orthogonal, overlapping, narrow band sub-channels or 
subcarriers, transmitted in parallel, divide the available transmission bandwidth. The separation of the subcarriers is theoretically optimal such that there is a very compact spectral utilization.

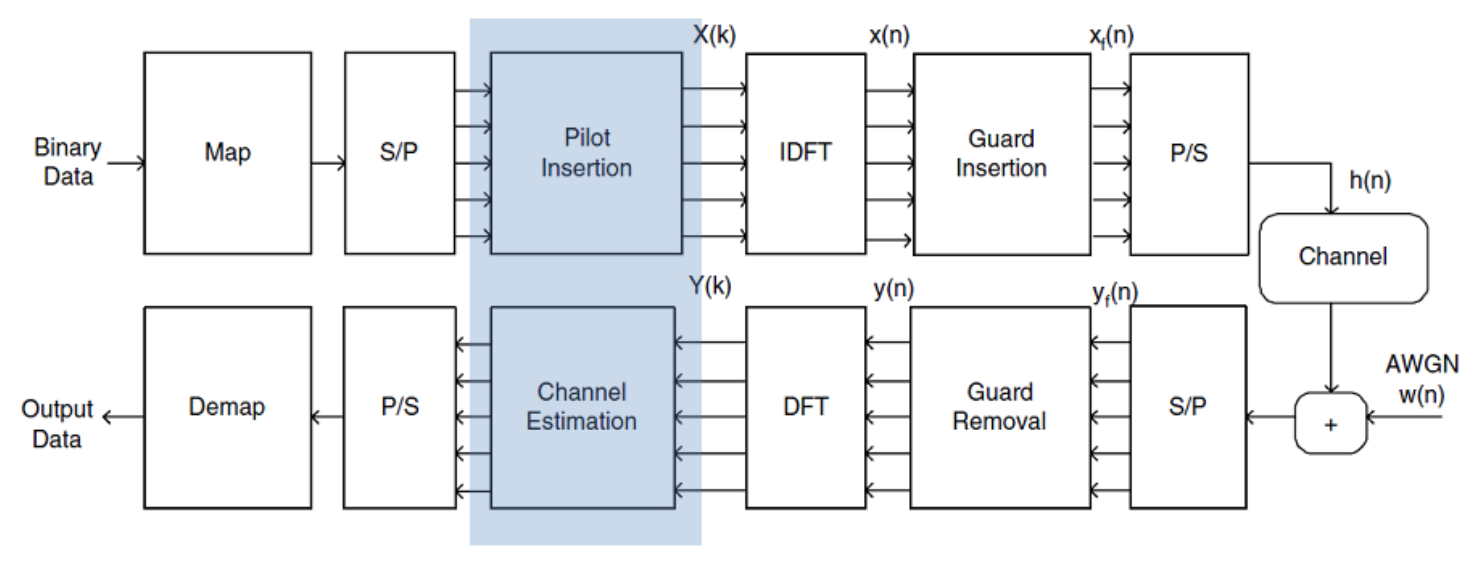

Figure 1. OFDM block diagram

Channel Estimation is the process of characterizing the effect of the physical medium on the input sequence. It is an important and necessary function for wireless systems. Even with a limited knowledge of the wireless channel properties, a receiver can gain insight into the data sent over by the transmitter. The main goal of Channel Estimation is to measure the effects of the channel on known or partially known set of transmissions. Orthogonal Frequency division multiplexing (OFDM) Systems are especially suited for channel estimation. The subcarriers are closely spaced. While the system is generally used in high speed applications that are capable of computing channel estimates with minimum delay.

Although ISI can be avoided, via the use of cyclic prefix in OFDM modulation, the phase and gain of each sub channel is needed for coherent symbol detection. An estimate of these parameters can be obtained with pilot/training symbols, at the expense of bandwidth. Blind channel estimation methods avoid the use of pilot symbols, which makes them good candidates for achieving high spectral-efficiency. Existing blind channel estimation methods for OFDM systems can be classified as Statistical \& Deterministic.

The statistical methods explore the cyclostationarity that the cyclic prefix induces to the transmitted signal. They recover the channel using cyclic statistics of the received signal, or subspace decomposition of the correlation matrix of the pre-DFT received blocks. The deterministic methods process the post DFT received blocks, and exploit the finite alphabet property of the information bearing symbols. Maximum likelihood and iterative Bayesian methods are two examples. Taking into account, specific properties of MPSK or QAM signals, while utilizing an exhaustive search. In comparison to the statistical methods, the deterministic ones converge much faster, however, they involve high complexity, which becomes even higher as the constellation order increases.

The work for the Blind channel detection and estimation started by using fast blind trellis search techniques [1] over linear distortive. This relied on the principle of finding the best possible channel fit for a noisy channel output sequence but this technique suffered fro a shortcoming of non instrument able due to the exponential growth in its complexity with data but has the feature of fast convergence. The proposed algorithm analyzed and compared between trellis decoding and channel estimation algorithm using Recursive least squares or LMS. Further refinements were made by [2] which analyzed the blind identification by determining FIR parameters from systems output which were excited from discreet alphabets.

This approach utilized the discrete alphabet property of applied inputs and has an advantage of robustness to noise structure but sensitive to noise level. It yielded exact impulse response for noise less systems. This work on blind channel estimation also concluded the dependence of convergence time on system order and size of input alphabet. Also the proposed algorithm showed insensitivity to noise probability distribution. In [3] Maximum likelihood approach was used in combination with self adaptive technique and this new technique overcome the problem of estimating the most likely state sequence of discreet time finite state Markov process with known parameters. This ML scheme is for both input sequences and parameters for estimation of state sequences. This work had also the feature of asymptotically differing from the conventional MLSE with known coefficients having low probability.

Further research in this direction was estimation using blind trellis search techniques [4]. It covered the ML estimation fro data transmitted over unknown linear channels without any prior requirement for the 
initial sequence as required by earlier conventional methods. Simultaneously both channel and data are estimated by obtaining least square channel estimate between received and data sequence and henceforth selection of the optimal. Complexity of this algorithm was higher than the viterbi algorithm for more than two alphabets. It also led to the convergence over a wide range of SNR within 100 symbols. A step ahead was taken in this direction by Blind estimation of multiple co channel digital signals by utilizing antenna array [5].This work had an extra feature of separating and estimating multiple co channel digital signals. It utilized the temporal structure of digital signals for determining array response and bit sequence for each signal.

Another novel approach for blind identification of Multichannel systems was taking FIR filters which utilized the concept of orthogonality between signals and noise sub space for creating a quadratic form which after minimization yields desired estimates. This work had some certain advantages over the previous works and technologies proposed as it being computationally efficient is have no apriori requirement of emitted symbol correlation. This method has advantage over the previous that it leads to precise estimates of channel coefficients for short data frame and used for fast convergence. Another approach in the same direction was made by [7] for synchronous co channel digital signals using antenna array. This work takes into account ILSE and ILSP and concludes that ILSE converges to a fixed point with finite number of iterations. It can be also utilized for asynchronous transmission and multipath channels with large delay spread.

Besides exploring the methods for blind separation this work [8] also highlighted the semi blind methods for estimation of channels. Being a subspace based approach it utilized the cyclic prefix and has advantage of maintaining the classical OFDM based on CP insertion which makes it applicable to all standard multicarrier systems .It is very precise in cases where channel frequency response has no zeros located on a subcarrier. Another approach [9] of Blind channel estimation was also followed in the same year which utilized the concept of Cyclostationarity as an alternative to fractional sampling for blind channel estimation in OFDM systems. Here even channels with equi spaced unit circle zeros are identifiable in presence of any non zero CP.Also it highlighted that the impulse response shortening varies with channel and can be altered by changing the shortening parameters.

All the previous works assumed a perfect Channel state Information (CSI) to extract symbol estimates which resulted in a loss of performance which was not optimal hence an iterative Maximum likelihood sequential estimation was used [10] which used an EM (Expectation maximization) approach which converges rapidly for quasi static and non static fading channels while performance was similar to NL approach with perfect CSI. In the further course of time the ML approach of detection and decoding was extended to various Interference conditions [11] and information transfer from STC detector to an error correcting channel decoder. All the previous works assumed the channels to be stochastic but this work make itself distinct by conclusion of a deterministic Channel model which has advantages in receiver design by developing interference resistant algorithms.

As mentioned above the approach of Space time codes was analyzed using the Trained, Blind and semi blind detection schemes [12]. It compared and considered three estimation and detection schemes and obtained a new blind scheme without pilot symbol transmission for channel estimation. Assuming an I.I.D Gaussian random variable with zero mean and unit variance it concluded that blind detector is worse than semi blind as only one symbol was used for extracting information but for large values of block and small SNR blind detector performed better. Also BER of semi blind was found to be better then trained detector. Further taking forward the approach based on semi blind channel Identification and equalization [13] by the use of sparsity, maximum delay spread and apriori statistical information imposed by channel I addition with the pilot, cyclic prefix and finite alphabet constraints of transmitter required to reduce number of pilots needed fro channel and data recovery receiver utilizes the pilots for estimation.

Focusing more towards Blind estimation schemes low complexity Blind frequency offset estimation for OFDM over ISI channels [14].Prior to this work all CFO estimation techniques rely on assumption of CP greater than the channel. This work has the relevance due to its feature of low complexity and channel utilization by decreasing the length of CP. Another approach of Blind and semi blind channel estimation[15] explored the idea of sub space based estimation .It utilized the concept of redundancy introduced by cyclic prefix for channel identification and the best part of this method was that its compatibility with existing OFDM systems. Also it can work in completely blind approach without any initialization. But this algorithm has its limitation in terms of incapability to ensure the distinctness of channel estimation until complete noise subspace is considered.

Another approach for blind channel estimation followed the approach of second order cyclostationarity statistics [16] which utilized the pre coding and yielding estimates of channel. The performance of the estimation was independent of the structure of noise but it does require an apriori information on the upper bound of channel length. Another subspace based approach for blind channel 
estimation by using virtual carriers [17] which can be also used for existing OFDM systems with and without CP time dispersive channel. As stated in [14] the reduction in the length of CP leads to higher channel utilization than previous estimators, estimation accuracy and speed of convergence.

This further research on blind channel estimation analyzed the performance over rapidly varying mobile radio channels.[18] It utilized the ML based approach and avoids the use of second and higher order statistics to improve convergence .It also used a combination of modulation schemes with which absolute phase of channel transfer function can be resolved. As done earlier in [16] which made use of pre coding techniques the further work also aimed at blind channel OFDM estimation using simple linear pre coding[20] where a simple transformation is applied on each block prior to its entry in OFDM system. This transform results in a correlation structure on transmitted blocks which are utilized at receiver for channel recovery It has the feature of fast convergence in addition to simplicity. This also outperforms the training based scheme used in IEEE 802.11a wireless standards.

In order to analyze and compare the performance of a blind channel with a semi blind channel scenario the work [21] made the estimates assuming a semi blind channel for channel response of multiuser antennas for a scalar matrix. For the number of users less than the number of symbols in pilot symbol block, the single pilot can remove ambiguity. The only requirement for estimation was an upper bound as in [16]for orders which are obtained from some apriori knowledge of propagation. In the same year another approach for blind channel estimation in combined sense by data detection and channel estimation via sphere decoding using ML principle. Over frequency selective fading channels. Here both V-Blast [22] and sphere decoding were used and can be also used for MIMO OFDM systems for fast fading channels.

In the next few years another work [23] on Blind maximum likelihood detection of orthogonal space time block codes was performed which concerns the problem of unknown CSI( channel state information on binary and quaternary PSK constellations. It utilized two approaches SDR (semi definite approach) which leads to a suboptimal but accurate blind ML detection. By using sphere decoding an exact ML blind detection algorithm was developed but its was computationally expensive.SDR approach leads to better results than sphere decoding in worst case. Under I.I.D. Rayleigh distribution stochastic blind ML Orthogonal space time block codes is equivalent to deterministic Blind ML OSTBC detector.

Another approach for blind channel estimation foe fractionally sampled FIR Channels [24] came into existence in the same phase which used the concept of residue polynomials for FIR channels. If received signal is mixed with inverse of transmitted signal the resulting transform renders channel transfer function in absence of additive noise. For an FIR samples of recovered impulse response must be zero in zeros region of channel impulse response. It was found to be more efficient than subspace based as it is deterministic and no requirement of auto correlation of received signal.

Further moving on lines of blind channel estimation the approach was made in the direction of Blind adaptive [25] estimation. It adopted the principle of zero padding OFDM and blind adaptive algorithm for finding impulse response of multipath signal. It uses RLS and LMS for obtaining fast convergence rate with minimum complexity. Both RLS and LMS were used for modification of orthogonal iteration for determining singular vectors.

The methodology of Blind channel estimation using cyclic prefixed single carrier systems using real symbol characteristics [26]. The property of second order statistics which were present in the transmitted data block. It was a simple method using virtual carriers or redundant coding resulting in bandwidth efficiency. also another distinguishing feature of this approach was reduction in phase ambiguity and which is converted to symbol ambiguity only. It was generally preferred for SISO but can be extended to SIMO and MIMO. In the same phase the blind ML detection of OSTBC [27] was performed with emphasis on binary PSK and QPSK. This work also classified a category called non rotatable OSTBC which were known as UIUTSUniquely identified up to a sign almost certainly with few assumptions. For an Independently distributed Rayleigh with any number of receiver antennas, a non rotatable OSTBC can be UIUTS with unit probability.

For the case of MIMO OFDM Blind channel estimation [28] by combining the two systems of MIMO with the OFDM high data rates can be achieved over broadband wireless channels. It integrates and generalizes the existing subspace methods for blind channel estimation in SISO OFDM to estimation for two different MIMO OFDM distributions according to the number of transmitting and receiving antennas and can be also applied to MIMO OFDM without CP regardless of the presence of virtual carrier hence improving the transmission efficiency.

Further approaches for blind channel estimation for MIMO OFDM systems by the use of non redundant linear pre coding [29] was analyzed by assuming transmitted symbols to be I.I.D. considering a subspace based approach. It can be efficiently used where number of receiving antennas is less than transmitting antenna i.e. MISO using second order statistical analysis. Further extension of the work carried in [29] was extended in [30] for non redundant pre coding for Semi blind and Blind channel estimation. It considers the combination of MIMO with block transmission using cyclic prefix which results in high data 
rates and simplification of Channel estimation and equalization .This approach can be used in cases where number of transmitting are more than receiving and leads to improved MSE and BER by taking large value at low SNR and very small value at high SNR.

Another approach for MIMO OFDM systems dealt with the subspace based blind channel estimation with emphasis on short averaging periods[31].It again utilized the orthogonality property of the noise and signal subspaces by applying a signal noise space decomposition to correlation matrix of received signal. The work laid emphasis on desired correlation matrix by educing number of OFDM blocks for time averaging. It has fast convergence by utilizing the frequency correlation between adjacent subcarrier by subcarrier groupings.

Working on the same lines of Blind channel estimation a need for robust subspace method was felt for CP MIMO OFDM [32] which used the re modulation on received cannel blocks. The most promising part of this work was its compatibility with existing as well the future 4G based communication systems. Another approach of cholesky factorization makes the covariance matrix a special structure for channel estimation for blind FIR channel identification [33].It is used for small samples and computationally efficient. Blind Maximum likelihood detection for SIMO systems with low complexity [34] for general constellations proposed the use of a sequential decoder for exact joint ML solution shown by joint maximum likelihood channels estimation. As preprocessing required in Chloe sky or QR decomposition there is no such requirement. As SNR grows the complexity of the algorithm approaches a constant time data length.

Earlier methods of Blind ML detection relied on static nature of channels. The work considers semi blind ML detection of OSTBC OFDM with single blocks [35]. The main advantage of this methodology was to accommodate channels with short coherence time considering BPSK or QPSK implementation which reduced complexity by detection using sub channel grouping. Also in case of identifiability analysis it ensures a unit probability condition by using less number of pilots and tries to achieve large scale optimization. For orthogonally coded OFDM MIMO systems the blind channel estimation again used the SDR (semi definite relaxation) approach [36] as in [23] which uses the certain properties of OSTBC for estimation of FIR in time domain instead of frequency domain independently of each subcarrier. The Semi definite approach has advantages over conventional methods.

Further developments in sub space based blind channel estimation for OFDM MIMO systems [37] which again utilized the orthogonality of noise and signal spaces of correlation matrix of received signal. Besides orthogonality it also used the reduced time averaging using frequency correlation among adjacent carriers in MIMO OFDM and also required less number of time samples. Blind channel estimation approach came to a new platform by adopting an iteration based approach for OFDM systems [38].This approach differs from the previous approaches in the sense that instead of using pilots it makes primary estimates of data symbols for each subcarrier then these estimates are applied to optimal MMSE estimation which requires only one value of time frequency correlation of channel transfer function. As compared to known decision based Kalman estimation and two pilot aided OFDM schemes this scheme performs better from mid to high SNR range. And its performance shows small degradation for mismatching.

Blind channel estimation techniques were also analyzed in light of enhanced data recovery using cyclic prefix by using output symbol and cyclic prefix transmitted over block fading channel. This work[39] proposed the iterative methods for reduction in complexity. This work also concluded that data recovery is possible only with output data irrespective of channel zeros locations for which Newton's method performed better for all values of SNR for moderately high number of carriers.

Taking into account all the previous works done on all the possible approaches for blind channel estimation this work [40] aimed at a low complexity blind equalization for OFDM systems with general constellations. This work also took into account the variation in channel on symbol by symbol basis which is suited for fast fading channels. This feature of the work made it distinct from all the previous works carried out for blind channel estimation as it takes in to account the symbol by symbol variation for fat fading channels. Complexity of this algorithm is low at high SNR and it recovers data from output observations only without any information of channel statistics hence the most reliable with respect to previous approaches.

\section{RESULTS AND ANALYSIS}

The work [40] carried considered an OFDM system with, or 64 subcarriers and a CP of length $\mathrm{L}=\mathrm{N} / 4$. The uncoded data symbols are modulated using BPSK, 4-QAM, or 16-QAM. The constructed OFDM signal then passes through a channel of length ,which is assumed to be block fading (i.e., constant over one OFDM symbol but fades independently from one symbol to another) and whose taps follow an exponential decay profile, Comparison of the performance of algorithm was made against the following receivers: the subspace-based blind receiver, the sphere decoding based receiver, a receiver that acquires the 
channel through training with pilots and a priori channel correlation, the ML receiver that acquires data through exhaustive search. The simulations were averaged over 500 Monte-Carlo runs.

Figure 2 compares the BER performance of algorithm with the aforementioned algorithms for an OFDM system with subcarriers and BPSK data symbols. In work the blind algorithm outperforms both the subspace and sphere decoding algorithms and almost matches the performance of the exhaustive search algorithm for low and high SNR, which confirms the ML nature of the algorithm. Figure 3, which considers the 4-QAM case, shows the same trends observed for the BPSK case of Figure 2.

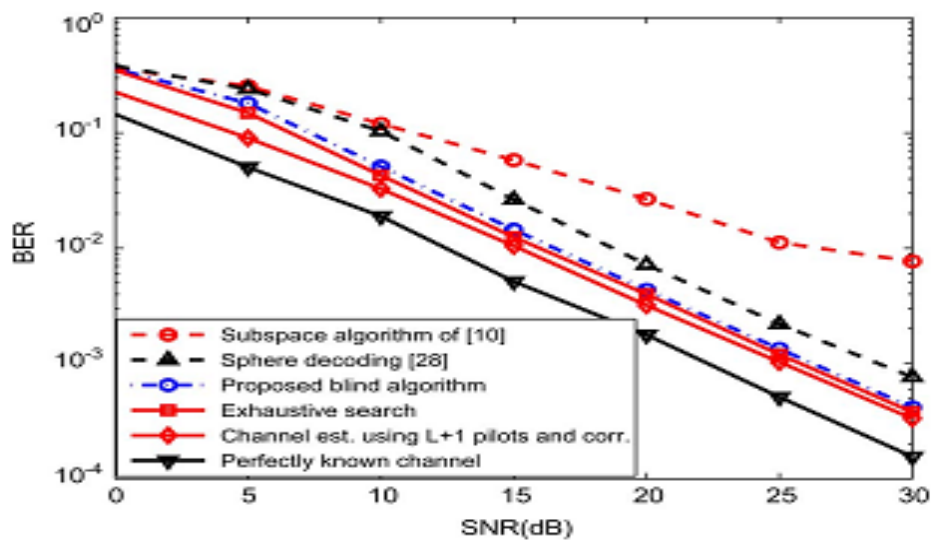

Figure 2. BER vs SNR for BPSK OFDM over a Rayleigh channel with and L=3

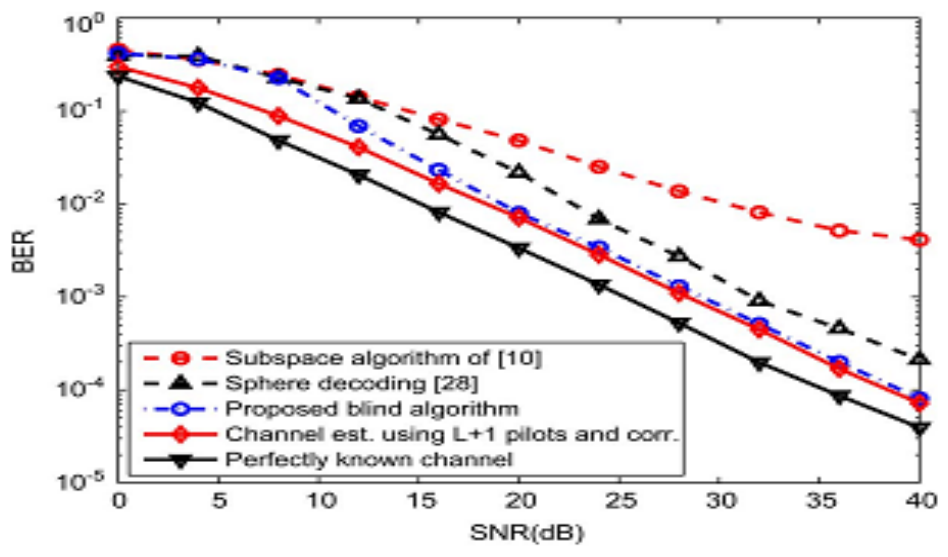

Figure 3. BER vs SNR for 4-QAM OFDM over a Rayleigh channel with N=16 and L=3

Figure 4 considers a more realistic OFDM symbol length, symbols drawn from a 4-QAM constellation and allows the SNR to grow to $45 \mathrm{~dB}$. The proposed blind algorithm [40] shows no error, which is characteristic of non-ML methods. Furthermore, the algorithm beats the training-based method and follows closely the performance of the perfect channel knowledge case. Figure 5 shows the results with subcarriers and 16-QAM data symbols for SNR as large as $50 \mathrm{~dB}$. 


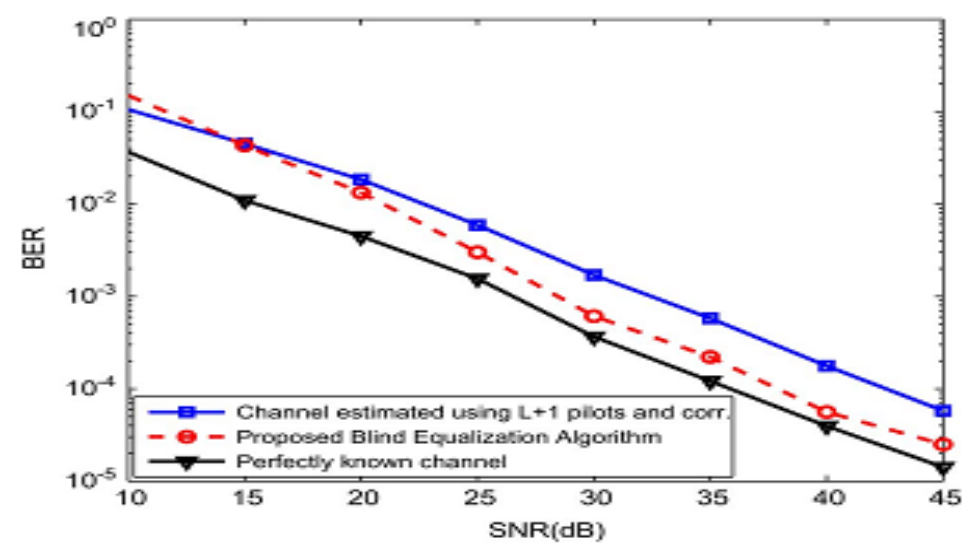

Figure 4. BER vs SNR for 4-QAM OFDM over a Rayleigh channel with N=64 and L=15

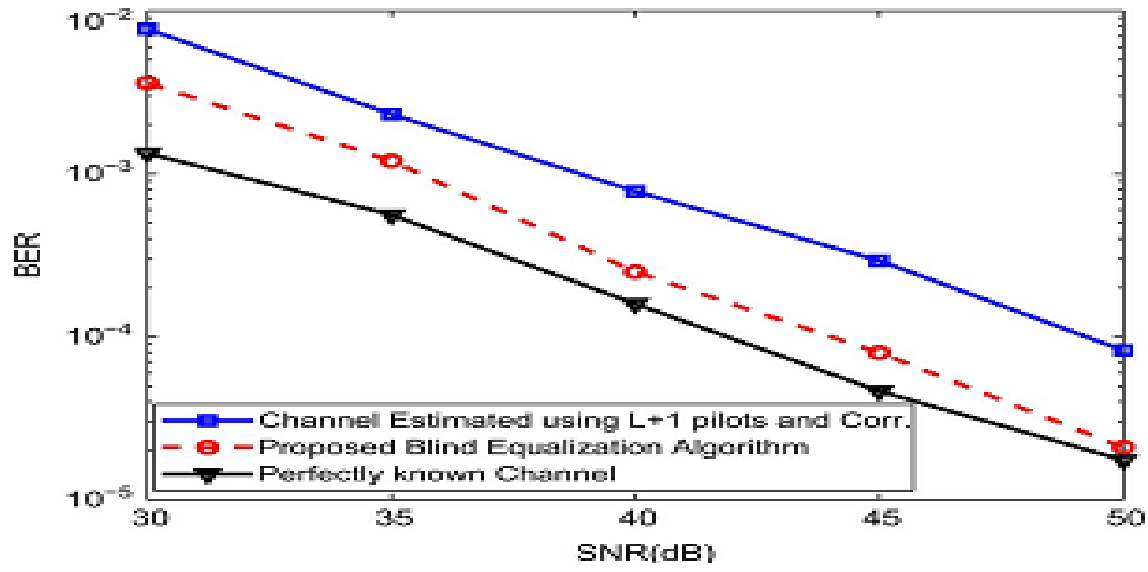

Figure 5. BER vs SNR for 16-QAM OFDM over a Rayleigh channel with N=64 and L=15

\section{CONCLUSION}

In this paper several works on blind channel estimation and equalization have been analyzed. The approaches on blind channel estimation can also be broadly classified into maximum-likelihood (ML) methods and non-ML methods. The non-ML methods include approaches based on subspace techniques, second-order statistics, Cholesky factorization, iterative methods, virtual carriers, real signal characteristics and linear pre coding. The paper analyzed that subspace-based methods have lower complexity, but suffer from slow convergence as they require many OFDM symbols to provide an accurate estimate of the channel autocorrelation matrix. Whereas Blind methods based on second-order statistics also require the channel to be strictly stationary over several OFDM blocks. Methods based on Cholesky factorization and iterative techniques have demerit in terms of high computational complexity. These features make these algorithms suitable for block fading with short channel coherence times. Generally suboptimal approximations are utilized for reduction of the computational complexity of ML-based methods. These methods reduce the complexity of the exhaustive ML search, they still results in high computational cost. A few ML-based algorithms allow the channel to change on a symbol-by-symbol basis [40] which has an advantage in terms of a low-complexity by utilizing the structural features of the partial fast Fourier transform (FFT) matrices and aimed at complexity minimization in the high SNR regime.

\section{REFERENCES}

[1] N. Seshadri, “Joint data and channel estimation using blind trellis search techniques ,"IEEE Trans. Communication., vol. 42, pp. 1000-1011,Feb.- Apr. 1994.

[2] D. Yellin and B. Porat, "Blind identification of FIR systems excited by discrete-alphabet inputs,”IEEE Trans. Signal Process., vol. 41, pp.1331-1339, Mar. 1993

[3] B. P. Paris, "Self-adaptive maximum-likelihood sequence estimation, “in Proc. IEEE GLOBECOM, 1993. 
[4] N. Seshadri, "Joint data and channel estimation using blind trellis search techniques ,"IEEE Trans. Commun., vol. 42, pp. 1000-1011,Feb.- Apr. 1994.

[5] S. Talwar, M. Viberg, and A. Paulraj, "Blind estimation of multiple co-channel digital signals using an antenna array,” IEEE Signal Process. Lett., vol. 1, pp. 29-31, Feb. 1994

[6] E. Moulines, P. Duhamel, J. F. Cardoso, and S. Mayrargue, "Subspace methods for the blind identification of multichannel FIRfilters,”IEEE Trans. Signal Process., vol. 43, no. 2, pp. 516-525, Feb. 1995.

[7] S. Talwar, M. Viberg, and A. Paulraj, "Blind estimation of multiple co-channel digital signals using an antenna array,” IEEE Signal Process. Lett., vol. 1, pp. 29-31, Feb. 1994.

[8] B. Muquet, M. de Courville, and P. Duhamel, "Subspace-based blind and semi-blind channel estimation for OFDM systems,”IEEE Trans.Signal Process., vol. 50, no. 7, pp. 1699-1712, Jul. 2002.

[9] R. W. Heath, Jr. and G. B. Giannakis, "Exploiting input cyclostationarity for blind channel identification in OFDM systems,”IEEE Trans.Signal Process., vol. 47, no. 3, pp. 848-856, Mar. 1999

[10] Y. Li, C. Georghiades, and G. Huang, "Iterative maximum likelihood sequence estimation for space-time coded systems,” IEEE Trans.Commun., vol. 49, no. 6, pp. 948-951, 2001

[11] E. Larsson, P. Stoica, and J. Li, "On maximum-likelihood detection and decoding for space-time coding systems," IEEE Trans. Signal Process., vol. 50, no. 4, pp. 937-944, 2002

[12] P. Stoica and G. Ganesan, "Space-time block codes: Trained, blind and semi-blind detection, ”Digital Signal Process., vol. 13, pp. 93-105,2003

[13] T. Y. Al-Naffouri, A. Bahai, and A. Paulraj, "Semi-blind channel identification and equalization in OFDM: An expectation-maximization approach,” in Proc. IEEE Veh. Technol. Conf., Vancouver, Canada, Sep.2002,vol.1,p

[14] Chengyang Li, Man-On Pun and Sumit Roy Department of Electrical Engineering, BOX 352500 University of Washington, Seattle, WA 98195-2500,” Low Complexity Blind Frequency-Offset Estimator for OFDM systems over IS1 channels ”, 0-7803- 632-3/02/ 02002 IEEE

[15] B. Muquet, M. de Courville, and P. Duhamel, "Subspace-based blind and semi-blind channel estimation for OFDM systems,”IEEE Trans. Trans. Signal Process., vol. 50, no. 1, pp. 96-109, Jan. 2002 Signal Process., vol. 50, no. 7, pp. 1699-1712, Jul. 2002.

[16] H.Bölcskei, R.W.Heath,Jr., andA.J.Paulraj,“Blind channel identification and equalization in OFDM-based multiantenna systems,"IEEE

[17] C. Li and S. Roy, "Subspace-based blind channel estimation for OFDM by exploiting virtual carriers, ”IEEE Trans. Wireless Commun.,vol.2,no. 1, pp. 141-150, Jan. 2003

[18] M. C. Necker and G. L. Stüber, “Totally blind channel estimation for OFDM on fast varying mobile radio channels, ’IEEE Trans. Wireless Commun., vol. 3, no. 5, pp. 1514-1525, Sep. 2004.

[19] A.GalloandE.C.F.M.Vitetta,"BEM-basedSISOdetectionoforthogonal space-time block codes over frequency flatfading channels, ”IEEE Trans. Wireless Commun., vol. 3, no. 6, pp. 1885-1889, 2004.

[20] A.Petropulu, R. Zhang, and R. Lin, "Blind OFDM channel estimation through simple linear precoding, ”IEEE Trans. Wireless Commun., vol.3, no. 2, pp. 647-655, Mar. 2004.

[21] Y. Zeng and T. S. Ng, "A semi-blind channel estimation method for multiuser multi user multiantenna OFDM systems,”IEEE Trans. Signal Process., vol. 52, no. 5, pp. 1419-1429, May 2004

[22] Tao Cui ; Dept. of Electr. \& Comput. Eng., Alberta Univ., Edmonton, Alta., Canada ; Tellambura, C.” Joint channel estimation and data detection for OFDM systems via sphere decoding" Global Telecommunications Conference, 2004. GLOBECOM '04. IEEE, ,3656 - 3660 Vol.6.

[23] W. Ma, B. Vo, T. Davidson, and P. Ching, "Blind ML detection of orthogonal space-time block codes: Efficient high-performance implementations, ”IEEE Trans. Signal Process., vol. 54, no. 2, pp. 738-751,2006.

[24] H. Muarkami, "Blind estimation of a fractionally sampled FIR channel for OFDM transmission using residue polynomials,” IEEE Trans.Signal Process., vol. 54, no. 1, pp. 225-234, Jan. 2006.

[25] X. G. Doukopoulos and G. V. Moustakides, "Blind adaptive channel estimation in OFDM systems,”IEEE Trans. Wireless Commun.,vol.5,no. 7, pp. 1716-1725, Jul. 2006

[26] F. Gao, A. Nallanathan, and C. Tellambura, "Blind channel estimation for cyclic prefixed single-carrier systems by exploiting real symbol characteristics, ”IEEE Trans. Veh. Technol., vol. 56, no. 5, pp.2487-2498, Sep. 2007.

[27] W.-K. Ma, "Blind ML detection of orthogonal space-time block codes :Identifiability and code construction, "IEEE Trans. Signal Process., vol. 55, no. 7, pp. 3312-3324, Jul. 2007.

[28] F. Gao and A. Nallanathan, "Blind channel estimation for MIMO OFDM systems via non redundant linear pre coding , ’IEEE Trans.Signal Process., vol. 55, no. 2, pp. 784-789, Feb. 2007.

[29] F. Gao and A. Nallanathan, "Blind channel estimation for MIMO OFDM systems via non redundant linear pre coding , ”IEEE Trans.Signal Process., vol. 55, no. 2, pp. 784-789, Feb. 2007.

[30] Changyong Shin, Member, IEEE, Robert W. Heath, Jr., Senior Member, IEEE, and Edward J. Powers, Fellow, IEEE,” Non-Redundant Precoding-Based Blind and Semi-Blind Channel Estimation for MIMO Block Transmission With a Cyclic Prefix”, IEEE Transactions On Signal Processing, Vol. 56, No. 6, June 2008

[31] C.-C. Tu and B. Champagne, "Subspace blind MIMO-OFDM channel estimation with short averaging periods: Performance analysis,” in Proc. IEEE Wireless Commun. Netw. Conf.,LasVegas,NV,Apr.2008, pp. 24-29.

[32] F.Gao,Y.Zeng,A.Nallanathan,andT.S.Ng, "Robust subspace blind channel estimation for cyclic prefixed MIMO OFDM systems,”IEEE J. Sel. Areas Commun., vol. 26, no. 2, pp. 378-388, Feb. 2008.

[33] J. Choi and C. C. Lim, “Cholesky factorization based approach for blind FIR channel identification ,"IEEE Trans. Signal Process., vol.56, no. 4, pp. 1730-1735, Apr. 2008. 
[34] W. Xu, M. Stojnic, and B. Hassibi, "Low-complexity blind maximum likelihood detection for SIMO systems with general constellation,” in Proc. IEEE Int. Conf. on Acoust., Speech, Signal Process. (ICASSP),Las Vegas, NV, Apr. 2008, vol. 1, pp. 2817-2820.

[35] T.-H. Chang, W.-K. Ma, and C.-Y. Chi, "Maximum-likelihood detection of orthogonal space-time block coded OFDM in unknown block fading channels,” IEEE Trans. Signal Process., vol. 56, no. 4, pp.1637-1649, Apr. 2008.

[36] N. Sarmadi, S. Shahbazpanahi,andA.B.Greshman, "Blind channel estimation in orthogonally coded MIMO-OFDM systems,”IEEE Trans.Signal Process., vol. 57, no. 6, pp. 2354-2364, Jun. 2009.

[37] C.-C. Tu and B. Champagne, "Subspace-based blind channel estimation for MIMO-OFDM systems with reduced time averaging,” IEEETrans. Veh. Technol., vol. 59, no. 3, pp. 1539-1544, Mar. 2010.

[38] S. A. Banani and R. G. Vaughan, "OFDM with iterative blind channel estimation, ”IEEE Trans. Veh. Technol., vol. 59, no. 9, Nov. 2010.

[39] T. Y. Al-Naffouri and A. A. Quadeer, “Cyclic prefix based enhanced data recovery in OFDM,”IEEE Trans. Signal Process., vol. 58, no. 6,pp. 3406-3410, Jun. 2010.

[40] Tareq Y. Al-Naffouri, Member, IEEE, Ala A. Dahman, Muhammad S. Sohail, Weiyu Xu, Member, IEEE,and Babak Hassibi, Member, IEEE “Low-Complexity Blind Equalization for OFDM Systems With General Constellations" IEEE Transactions On Signal Processing, Vol. 60, No. 12, December 2012.

[41] Surinder Singh,Sandeep Singh Gill,” Performance Evaluation of Channel Estimation in OFDM System for

[42] Different QAM and PSK Modulations”, International Journal of Electrical and Computer Engineering (IJECE),Vol 1 No 2, 2011 pages 140-150.

[43] Anis Charrada, Tunisia,” Support Vector Machines Regression for MIMOOFDM Channel Estimation”, IAES International Journal of Artificial Intelligence (IJ-AI),Vol 1 No 4, 2012 pages 214-224.

\section{BIOGRAPHIES OF AUTHORS}

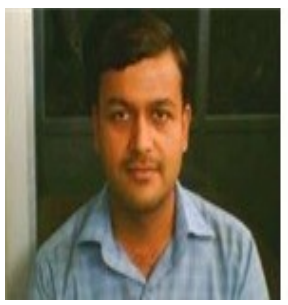

Mr.Vivek Kumar Gupta,Research received B.Tech Degree from CCSU university,after that he received his M.Tech degree from Uttarakhand technical university,Dehradun,Uttarakhand.

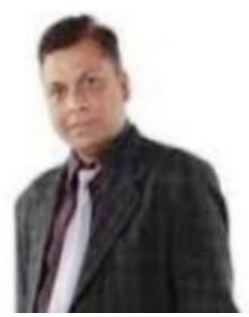

Dr.Sandip Vijay is an eminent Professor of ICFAI University and has guided many scholars in Ph.d,also he has publication in many international journals of repute. 